\title{
Kurt J. Lesker
}

MRS Booth 301

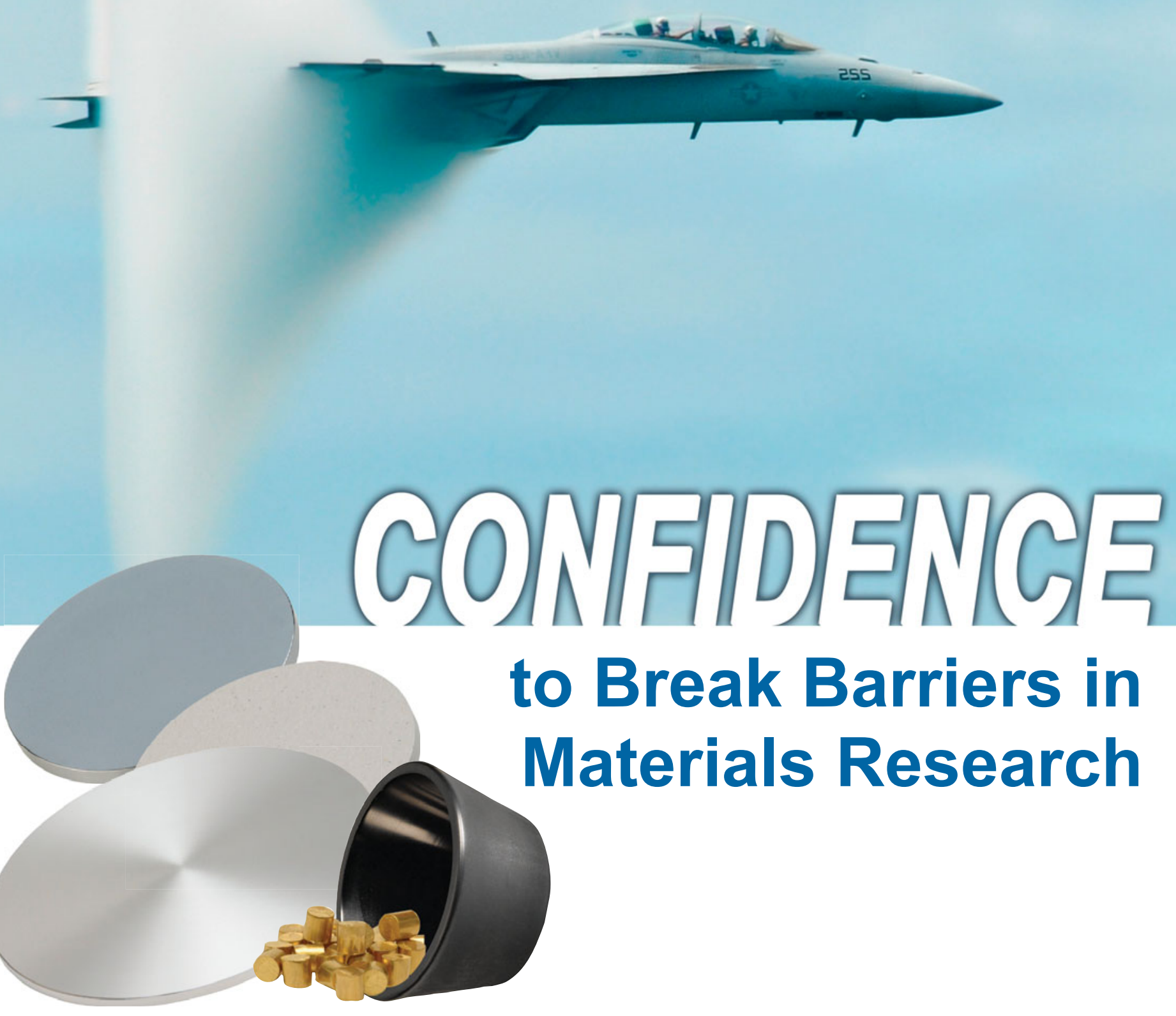

In-House Indium Bonding Service

Precious Metal Reclaim Program Customized Compositions and Stoichiometries Unmatched Technical and Application Support Extensive Inventory
Sputtering Targets, Evaporation Pellets, Pieces and Wire

Full Line of Sources for Thermal and E-beam Evaporation

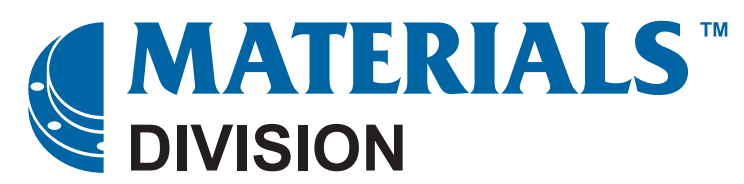



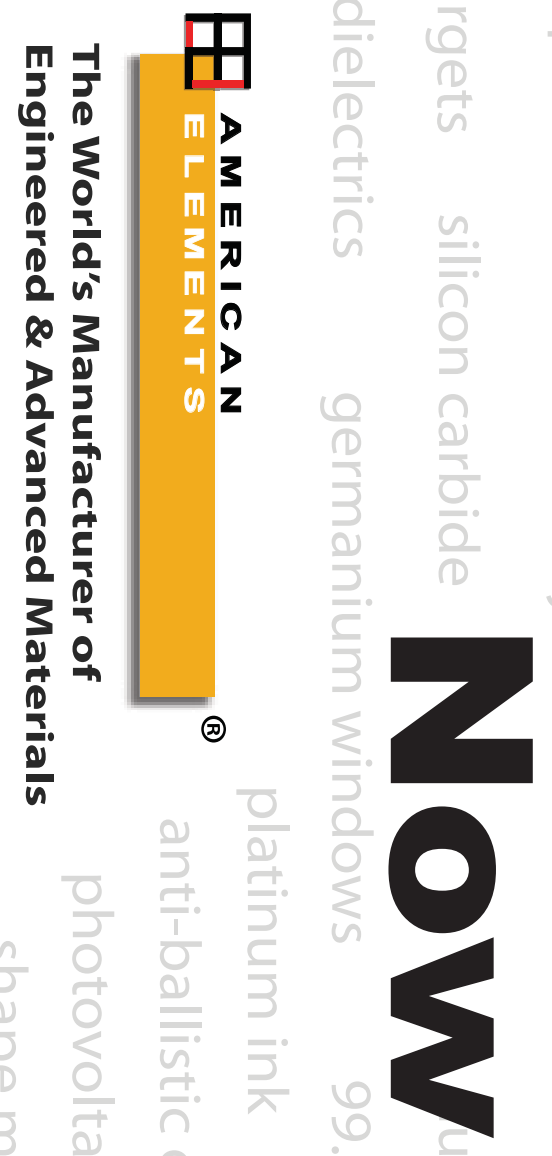

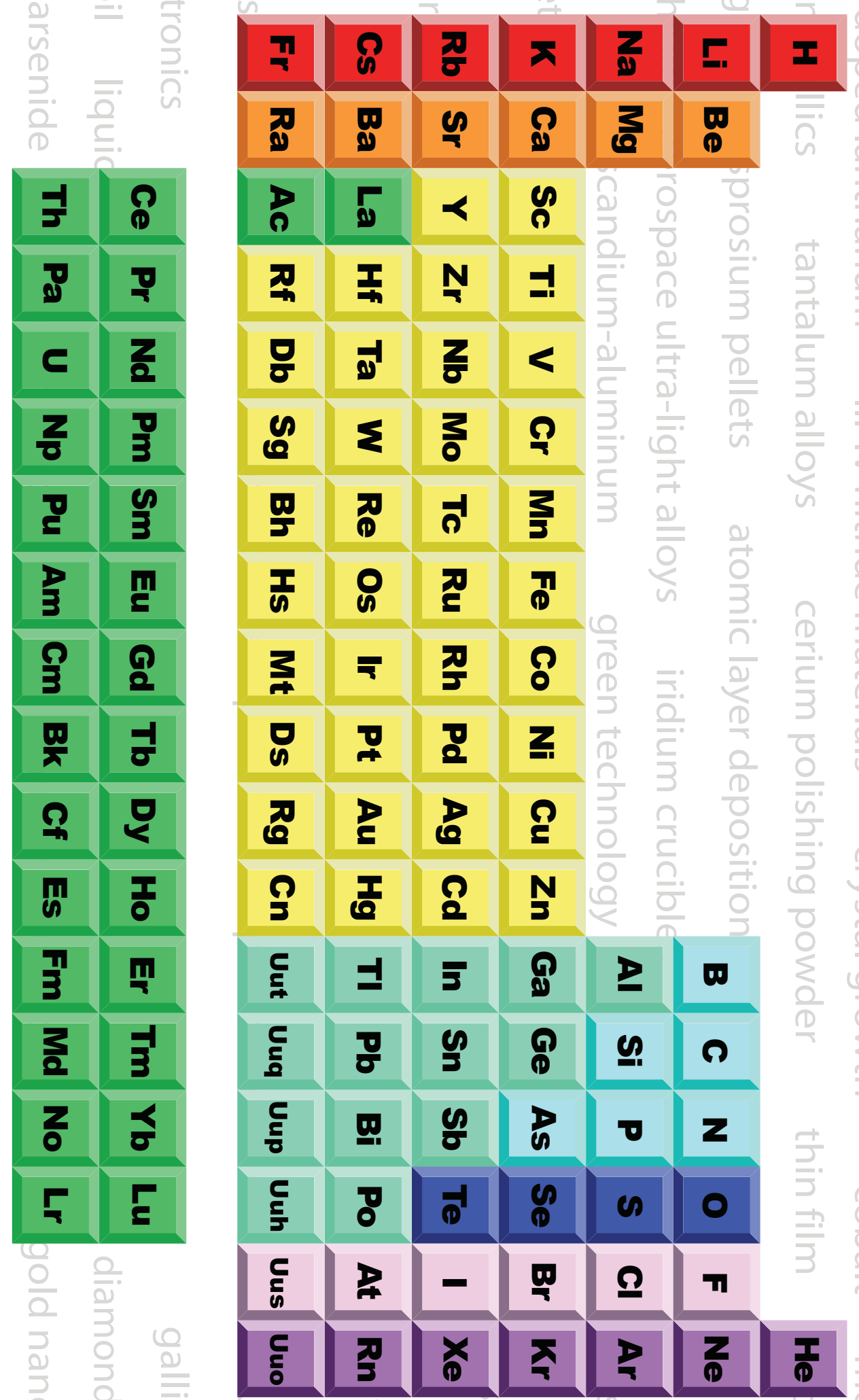

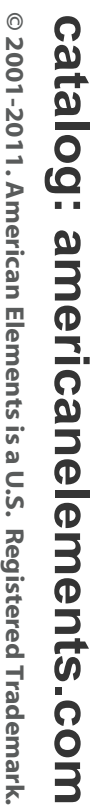
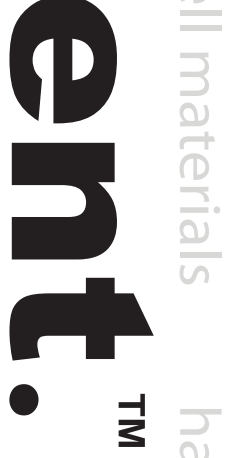